\title{
Specific Contact Resistivity Measurement by a Vertical Kelvin Test Structure
}

\author{
TAN FU LEI, LEN-YI LEU, AND CHUNG LEN LEE
}

\begin{abstract}
A vertical Kelvin test structure is used to measure the specific contact resistivity of the $\mathrm{AI}$ (1-percent $\mathrm{Si}$ ) / Si and $\mathrm{Al}$ (1-percent $\mathrm{Si}) / \mathrm{TiSi}_{2}$ / Si contact system. For the vertical test structure, the driving current flows "vertically," thus the current crowdings and sheet resistance effects are eliminated and measurement on the true sfecific contact resistivity becomes possible. Experimental works show that results obtained by using this vertical structure are closer to thi true specific contact resistivities than those obtained by using the conventional six-terminal Kelvin method. It is also found that $R_{s d}$, the sheet resistance directly underneath the contact pad, is much less than that of the conduction bar without the contact pad. A value of $(1.17 \pm$ $0.17) \times 10^{-7} \Omega \cdot \mathrm{cm}^{2}$ specific contact resistivity is obtained $\mathrm{for}$ the $\mathrm{Al}$ (1-percent $\mathrm{Si}) / \mathrm{TiSi}_{2}$ (direct reaction) / $\mathrm{Si}$ system.
\end{abstract}

\section{INTRODUCTION}

SPECIFIC contact resistivity $\rho_{c}$, defined as $\left(\partial J / \partial V^{\prime}\right)^{-1}$ for $V \rightarrow 0$, is one of the most important parameters in studying interfacial properties of metallization systems. Due to the scalings of semiconductor devices in VLSI, the required specific contact resistivity on contact systems in VLSI is in the order of $10^{-6} \sim 10^{-7} \Omega \cdot \mathrm{cm}^{2}$ [1]. Various contact systems and processes [1]-[3] have been studied to give the contact resistance of the above onder. However, as the contact resistance is reduced, accurate measurement of the "true" specific contact resistivity becomes more difficult. Many parasitic effects that were negligible previously become significant in determi aing quantities of the above order.

In measuring the specific contact resistivity, various test structures and methods have been proposed and stucied. Among them are: the two-terminal method by Cox and Strack [4], the transfer length (TLM) by Shockley [5], the four-terminal Kelvin method by Cohen et al. [6], anc the six-terminal Kelvin method by Proctor et al. [7]. For the former two methods, large errors will result since the zontact resistance is obtained by calculating small differe aces of large terms. For the latter two Kelvin methods, nore accurate, if not exact, measurement of the end contact resistance $R_{e}$, or the contact resistance $R_{c}$, can be obtained. However, two recent numerical studies [8], [9] of the six-terminal method showed that, for these two types of test structures, the inherent lateral current crowdin!s ef-

Manuscript received September 23, 1986; revised January 26, 987. This work was supported by the Electronic Research and Service Onganization (ERSO), ITRI, Republic of China.

The authors are with the Institute of Electronics, National Chiao Tung University, Hsin Chu, Taiwan, Republic of China.

IEEE Log Number 8613910.

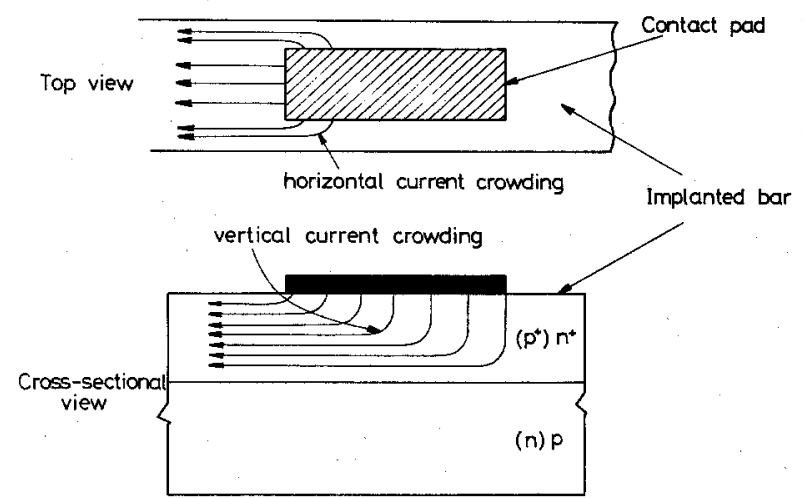

Fig. 1. A top view and a cross-sectional view of a "horizontal" type of test structure to show the horizontal crowding effect and the vertical crowding effect, respectively.

fects (both horizontal and vertical; see Fig. 1) and the junction sheet resistance effect affect the accuracy of the obtained contact resistance values. The reason for this inaccuracy comes from the fact that, for these two types of test structures, the current flows "horizontally" in a diffused bar while the "vertical" interfacial contact resistance is to be obtained.

In this paper, a "vertical" Kelvin test structure is presented to measure the "true" specific contact resistivity. For this test structure, the driving current flows "vertically" from the metal contact pad toward the contacted substrate. This eliminates the current crowding effects and the junction sheet resistance effect and makes the determination of the "true" specific contact resistivity possible. Besides, this test structure can be incorporated with the six-terminal test structure and the six-terminal measurement can be performed on the same contact. These two measurement results can be compared to ensure the correctness of measurements. Initial work on this test structure has been reported earlier [10].

This test structure is applied to Al (1-percent $\mathrm{Si}$ )/ $\mathrm{Si}$ and $\mathrm{Al}$ ( 1 percent $) / \mathrm{TiSi}_{2} / \mathrm{Si}$ contact systems to measure their contact resistances. All data show that this vertical test structure can give better measurement on the true specific contact resistivity. From measured values of specific contact resistivities and also the measured "end"' contact resistance from the same test structure $R_{s d}$, the sheet resistance underneath the contact pad is calculated: It is found that this sheet resistance is much less than that of the conduction bar. Also, a contact resistance of a value of $(1.17 \pm 0.17) \times 10^{-7} \Omega \cdot \mathrm{cm}^{2}$, measured by using 
this vertical test structure, has been obtained for the Al (1percent $\mathrm{Si}) / \mathrm{TiSi}_{2}$ (direct reaction) $/ \mathrm{Si}$ system.

\section{The Vertical Kelvin Test Structure}

Fig. 2(a) shows a cross-sectional view of the vertical Kelvin test structure and Fig. 2(b) shows its corresponding top view. Four masks are used to fabricate this structure. Mask 1 forms the isolation p-n junction. Mask 2 forms the heavily doped conduction bar. Mask 3 opens the contact window, and mask 4 defines the metallization pattern. Compared to the conventional four-terminal or six-terminal test structure, one extra mask step (mask 1) is needed. The driving current $I$ is forced from pad 1 toward the substrate and the voltage is sensed between pads 2 and 3 along the heavily doped conduction bar. The vertical current flow is restricted by an isolation p-n junction. For this structure, it can be seen that the current flows vertically and only through the contact window; hence, the current distribution in the contact region is metallurgically uniform. No vertical and horizontal current crowdings are expected. The contact resistance $R_{c}$ is thus directly measured with the value of $V / I$ and the specific contact resistivity is $A_{c}(V / I)$, where $A_{c}$ is the contact area through which the current passes. Also, the sheet resistance effect on determining the value of $\rho_{c}$ becomes minimum since it is not at all involved.

In Fig. 2, the six-terminal structure is also incorporated and this has two merits. First, the voltage $V$ can also be sensed between pad pairs $2-4,2-5$, and 2-6. The obtained values can be averaged with that of the pad pair 2 3 to reduce the error introduced by the experiments. Second, as mentioned previously, a six-terminal measurement can be performed on the same contact and the result obtained can be used to compare with that obtained by the vertical Kelvin method to assure the correctness of measurements. Furthermore, in this structure, the "end" contact resistance $R_{e}$ can also be measured directly. With this measured $R_{e}$, and the modified transmission line (MTL) model [11], the "front" contact resistance $R_{f}$ and the sheet resistance $R_{s d}$ of the heavily doped conduction bar underneath the contact region can be calculated through the following equations [11]:

$$
\begin{aligned}
R_{e}= & \left\{\left[\left(R_{s d} / W\right) / /\left(R_{s} / 2 \delta\right)\right] \times \rho_{c} / W\right\}^{1 / 2} / \\
& \sinh \left\{\left[\left(R_{s d} / W\right) / /\left(R_{s} / 2 \delta\right)\right]^{1 / 2} \times\left(W / \rho_{c}\right)^{1 / 2} \times d\right\}
\end{aligned}
$$

$$
\begin{aligned}
R_{f}= & \left\{\left[\left(R_{s d} / W\right) / /\left(R_{s} / 2 \delta\right)\right] \times \rho_{c} / W\right\}^{1 / 2} / \\
& \tanh \left\{\left[\left(R_{s d} / W\right) / /\left(R_{s} / 2 \delta\right)\right]^{1 / 2} \times\left(W / \rho_{c}\right)^{1 / 2} \times d\right\}
\end{aligned}
$$

where $A / / B=A B /(A+B), d$ and $W$ are the length and width of the contact region, and $\delta$ and $R_{s}$ are the alignment tolerance and sheet resistance of the conduction bar outside the contact region, respectively.

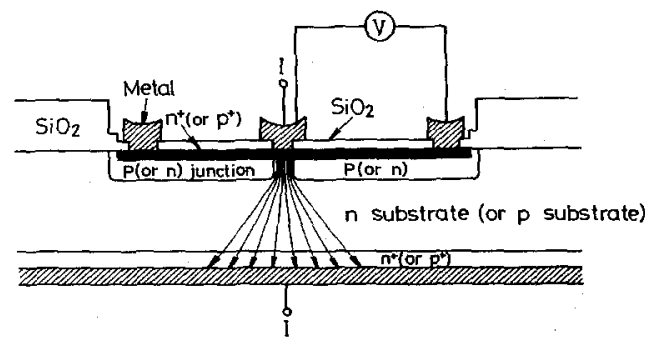

(a)

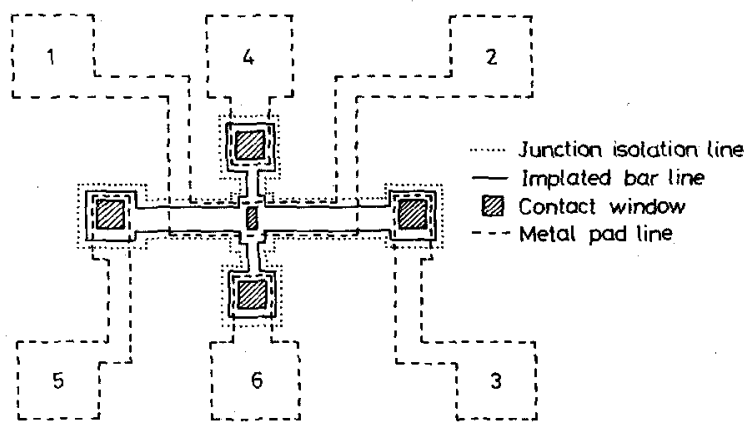

(b)

Fig. 2. (a) A cross-sectional view of the proposed vertical Kelvin test structure for measuring the true specific contact resistivity. (b) A top view of the structure. In the figure, the horizontal structure for the sixterminal measurement is also incorporated.

\section{EXPERIMENTS}

The test structure of Fig. 2 was fabricated to measure contact resistances. The test structures were formed on $\mathbf{n -}$ type substrates of resistivities of 3-5 $\Omega \cdot \mathrm{cm}$. For the measurement of sheet resistance of the conduction bar, the test patterns of the transfer length structure [1] were also formed on the same wafers. Two different contact system, i.e., $\mathrm{Al}$ (1-percent $\mathrm{Si}$ ) / Si and Al (1-percent $\mathrm{Si}$ )/ $\mathrm{TiSi}_{2} / \mathrm{Si}$ were used. For the vertical structure test patterns, eight contact sizes $(5 \mu \mathrm{m} \times 5 \mu \mathrm{m}, 5 \mu \mathrm{m} \times 10 \mu \mathrm{m}, 10 \mu \mathrm{m} \times$ $10 \mu \mathrm{m}, 10 \mu \mathrm{m} \times 15 \mu \mathrm{m}, 10 \mu \mathrm{m} \times 20 \mu \mathrm{m}, 15 \mu \mathrm{m} \times 15$ $\mu \mathrm{m}, 15 \mu \mathrm{m} \times 20 \mu \mathrm{m}$, and $20 \mu \mathrm{m} \times 20 \mu \mathrm{m}$ ) were used for the contact region and the alignment tolerance of the contacts was kept at $10 \mu \mathrm{m}$. A set of transfer length test patterns were also fabricated at the same time on the same wafer to measure the sheet resistance of the contact bar. For these transfer length test patterns, six contact windows, each $50 \mu \mathrm{m} \times 100 \mu \mathrm{m}$ in size and spaced apart from 20 to $60 \mu \mathrm{m}$ in $10-\mu \mathrm{m}$ increments, were used. The isolation $\mathrm{p}-\mathrm{n}$ junction was formed by ion implanting $\mathrm{BF}_{2}^{+}$with a dose of $2 \times 10^{13} / \mathrm{cm}^{2}$ and was kept shallow in the subsequent heat treatment to reduce the lateral diffusion. The junction of the $\mathbf{n}^{+}$heavily doped conduction bar was also kept shallow by ion-implanting $\mathrm{As}^{+}$to reduce the current spreading, which will contribute a measurement error to this test structure, in this region. In our experiments, the depths of the $\mathrm{n}^{+}$junctions were from 0.33 to $0.5 \mu \mathrm{m}$ for $2 \times 10^{15} / \mathrm{cm}^{2}$ to $6 \times 10^{15} / \mathrm{cm}^{2}$ ion doses of $\mathrm{As}^{+}$and the junction depths of the p-isolations were $0.7 \mu \mathrm{m}$ for a $2 \times 10^{13} / \mathrm{cm}^{2}$ ion dose of $\mathrm{BF}_{2}^{+}$. To form metallization contacts, for the $\mathrm{Al}$ (1-percent $\mathrm{Si}$ ) / $\mathrm{Si}$ 
system, Al(1-percent $\mathrm{Si}$ ) was vacuum-evaporated and deposited on the opened contact window. For the $\mathrm{Al}$ ( 1 percent $\mathrm{Si}$ ) $/ \mathrm{TiSi}_{2} / \mathrm{Si}$ system, two methods were used. One method was to coevaporate $\mathrm{Ti}$ and $\mathrm{Si}$ at two respective rates, and the formed film had a $\mathrm{Si} / \mathrm{Ti}$ ratio of 2 . T.1e film was then subjected to an annealing process [12] at $700^{\circ} \mathrm{C}$ for $15 \mathrm{~min}$ in an $\mathrm{H}_{2}$ ambient. The other method was to evaporate Ti first on wafers and followed by Mo and poly $\mathrm{Si}$ in sequence [13]. The formed multilayer structure was then annealed at $700^{\circ} \mathrm{C}$ in a $\mathrm{N}_{2}$ ambier $\mathrm{t}$. During annealing, Ti reacted with $\mathrm{Si}$ to form the $\mathrm{TiS}_{2}$ film and $\mathrm{Si} / \mathrm{Mo}$ acted as the protection layer for oxidation [14]. The $\mathrm{Si} / \mathrm{Mo}$ film was then stripped and $\mathrm{Al}$ (1-perce $1 \mathrm{t}$ $\mathrm{Si})$ was evaporated to form the $\mathrm{Al}$ (1-perce it $\mathrm{Si}) / \mathrm{TiSi}_{2} / \mathrm{Si}$ structure. For both methods, a sheet resi stance of $3 \Omega / \square$ was obtained for $700-\AA \mathrm{Ti}$ film. The back sides of wafers were $\mathbf{n}^{+}$diffused and $\mathrm{Al}$ metallized. In the measurement, a Keithley 230 programmable current source and a Keithley 619 programmable multimet:r were used to provide current driving and to sense voltages, respectively. For each wafer, at least 200 test patterns were measured.

\section{Experimental Results and Discussions}

For all the test structures fabricated, the $I-V$ characte: istics were linear at current levels from -5 to $0.5 \mathrm{~mA}$. All the contact resistances were measured within these current levels. Fig. 3(a) and (b) shows the log-log plots of the measured contact resistances for two wafers of the $\mathrm{Al}$ (1-percent $\mathrm{Si}$ )/Si contact system versus the contact area, where $R_{c v}$ and $R_{c s}$ are measured from the vertic 1 structure and the six-terminal structure, respectively. In these figures, straight lines obtained by the least squar fitting are also plotted for each set of data. In Fig. 3(a), the slope of the $R_{c v}$ straight line is -1.04 , while that of the $R_{c s}$ line is -0.88 . In Fig. 3(b), the $R_{c v}$ line has a slop: of -0.97 and the $R_{c S}$ has a slope of -0.95 . For an ideal contact, the slope should be -1 to satisfy the square lav [15]. For both figures, $R_{c v}$ values are closer to the ideal line. Fig. 4(a) and (b) shows similar plots for the contact system of $\mathrm{Al}$ (1-percent $\mathrm{Si}$ )/ $\mathrm{TiSi}_{2} / \mathrm{Si}$ formed by th: coevaporation method and by the direct reaction method, respectively. For both figures, the data points do not giv: good fits for straight lines. However, the slopes of the $R_{C}$ lines are always closer to -1 than those of $R_{c s}$ lines. They' are -0.89 and -0.82 for $R_{c v}$ and $R_{c s}$, respectively, itı Fig. 4(a) and -0.85 and -0.76 for $R_{c v}$ and $R_{c s}$, respec. tively, in Fig. 4(b). The reason for these large discrep ancies from the ideal value of -1 in these two figure mainly comes from the severe nonuniformity at the interface for the $\mathrm{TiSi}_{2} / \mathrm{Si}$ system. (The microscopy observa. tion on the contact area after stripping the contact metal revealed this.) The discrepancy from the ideal value ol the direct-reaction method is larger than that of the co. evaporation method. This result may be related to the fact that the interface of the former method is rougher thar that of the latter. In the above figures, it is also seen tha the $R_{c v}$ values are always smaller than the $R_{c s}$ values, and

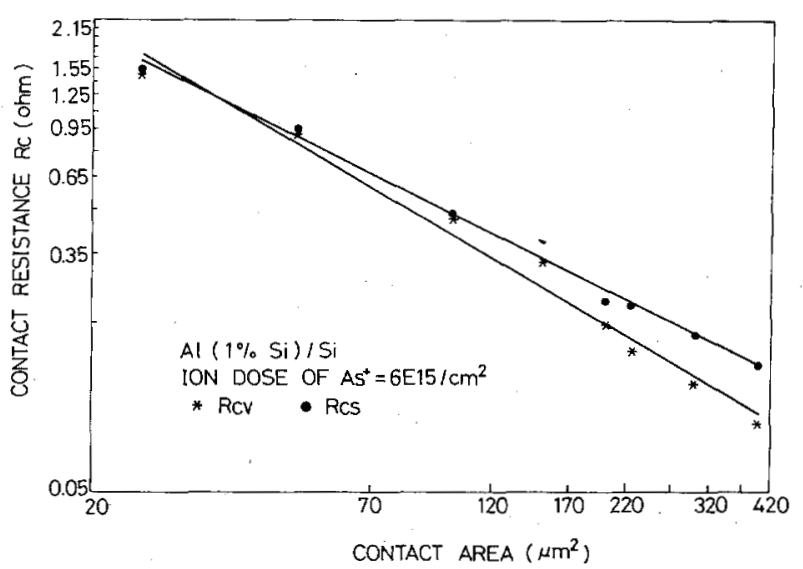

(a)

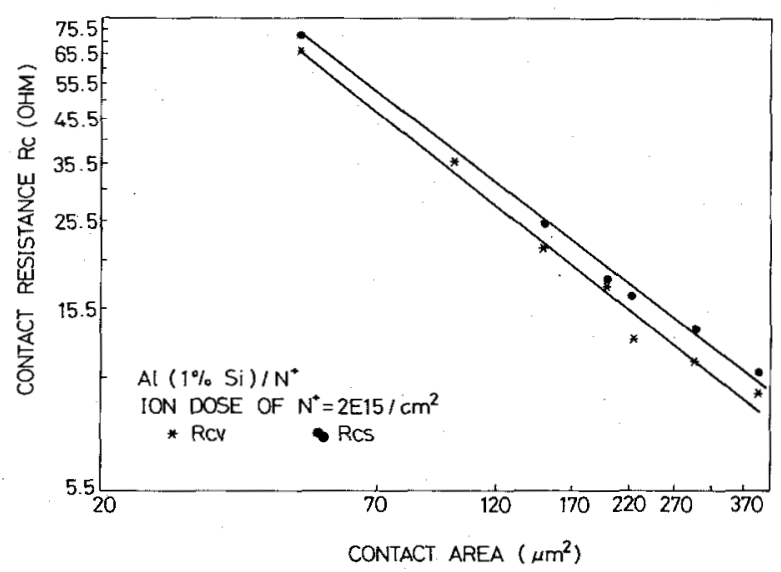

(b)

Fig. 3. The contact resistances of $\mathrm{Al}$ ( 1-percent $\mathrm{Si}$ )/ $\mathrm{Si}$ for (a) an ion dose of $\mathrm{As}^{+}=6 \times 10^{15} / \mathrm{cm}^{2}$ and (b) an ion dose of $\mathrm{As}^{+}=2 \times 10^{15} / \mathrm{cm}^{2}$ are plotted versus the contact area, where $R_{c v}$ and $R_{c s}$ were measured from the vertical structure and six-terminal structure. The straight lines are obtained by the least square fitting method for two sets of measured values, respectively.

the differences become larger for a larger contact area. This is expected since $R_{c v}$ values do not include the current crowding and the sheet resistance effects. For constant alignment tolerance of the contact, when the contact window is larger, these current crowding and sheet resistance effects become more serious. This phenomenon had also been predicted by [8].

The apparent specific contact resistivity of contact systems was measured with the vertical structure $\left(\rho_{c v}\right)$ and the six-terminal method $\left(\rho_{c s}\right)$ for keeping the contact size constant. Fig. 5(a) and (b) shows plots of $\rho_{c v}$ versus $\rho_{c s}$ with the contact areas being $20 \mu \mathrm{m} \times 20 \mu \mathrm{m}$ and $20 \mu \mathrm{m}$ $\times 15 \mu \mathrm{m}$, respectively. The various contact resistivities were obtained by implanting various doses of $\mathrm{As}^{+}$onto contact windows. For both figures, it is seen that $\rho_{c s} / \rho_{c v}$ deviates more from 1 for smaller specific contact resistivities. Since it has been predicted that $\rho_{c s}$ deviates more from the true value of $\rho_{c}$ as $\rho_{c}$ becomes smaller [9], this indicates that $\rho_{c v}$ does give the closer value to the true specific contact resistivity.

Since the end contact resistance $R_{e}$ can also be mea- 


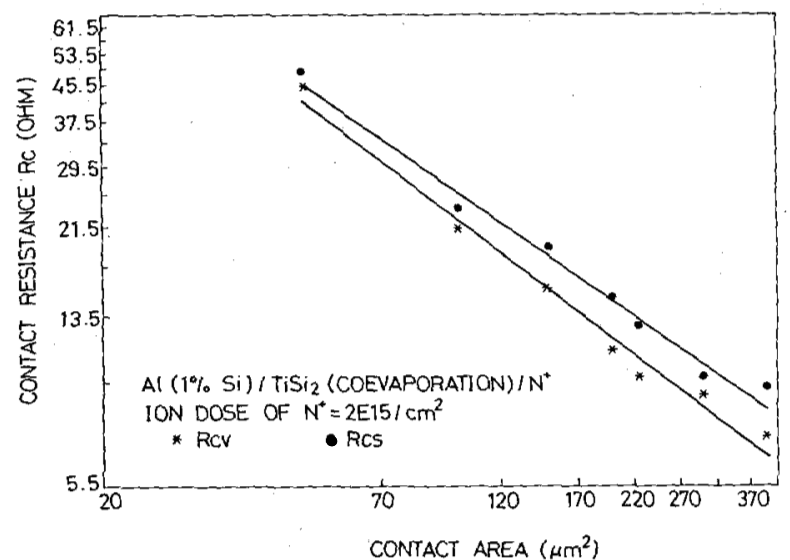

(a)

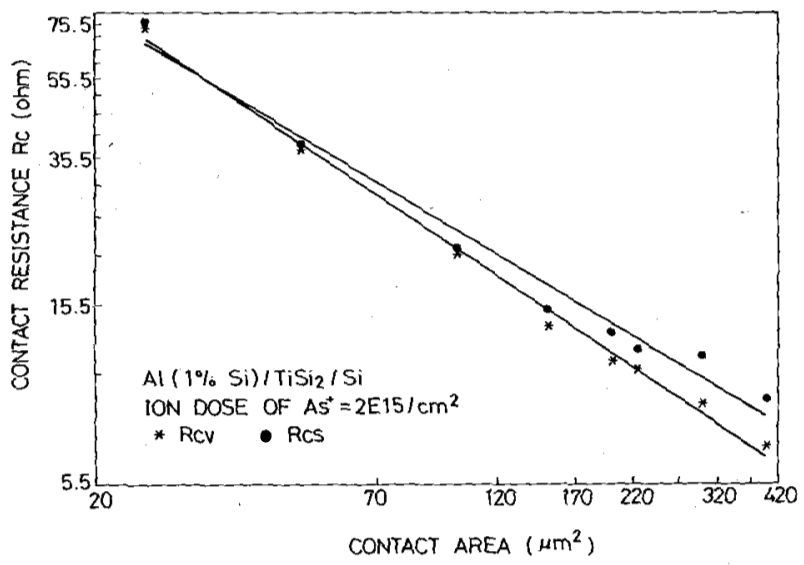

(b)

Fig. 4. The contact resistances of $\mathrm{Al}$ (1-percent $\mathrm{Si}$ ) $/ \mathrm{TiSi}_{2} / \mathrm{Si}$ for an ion dose of $\mathrm{As}^{+}=2 \times 10^{15} / \mathrm{cm}^{2}$ are plotted versus the contact area, where $\mathrm{TiSi}_{2}$ film was formed by (a) the coevaporation method and (b) the direct reaction method.

sured from the test structure of Fig. 2, it was measured along with $R_{c}$ for different contact areas. Fig. 6(a) shows the plots of the measured $R_{e}$ and $R_{c}$ values versus contact areas in linear scales for the $\mathrm{Al} /(1$-percent $\mathrm{Si}) / \mathrm{Si}$ system. In the figure, the experimental $R_{e}$ values were used to fit (1) to obtain the value for $R_{s d}$, the sheet resistance underneath the contact pad. In the fittings, $R_{s}$ was obtained from the separate TLM test structures that were fabricated on the same wafer. For this case, $R_{s}$ was obtained to be $46.5 \Omega / \square$ and the derived $R_{s d}$ value was 20.5 $\Omega / \square$. The fitted curve based on (1) for $R_{e}$ is plotted in the figure as the dotted curve. Also, the obtained $R_{s}$ and $R_{s d}$ values can be used to compute $R_{f}$ from (2). In the figure, it is plotted as the solid curve. It is seen that the $R_{f}$ curve is above the $R_{c}$ and $R_{e}$ curves in the figure, as it should be [11], [16]. It is worthwhile to mention that, in obtaining $R_{s d}$ from (1), if $\rho_{c s}$, instead of $\rho_{c v}$, was used for $\rho_{c}$, the errors in the least square fittings were much larger than that of the case when $\rho_{c v}$ was used. Fig. 6 (b) shows the fitted curves, for $\rho_{c s}$ cases with three $R_{s d}$ values. It is seen that even for $R_{s d}=46.5 \Omega / \square=R_{s}$, the computed $R_{e}$ curve is still above the measured $R_{c s}$ values.

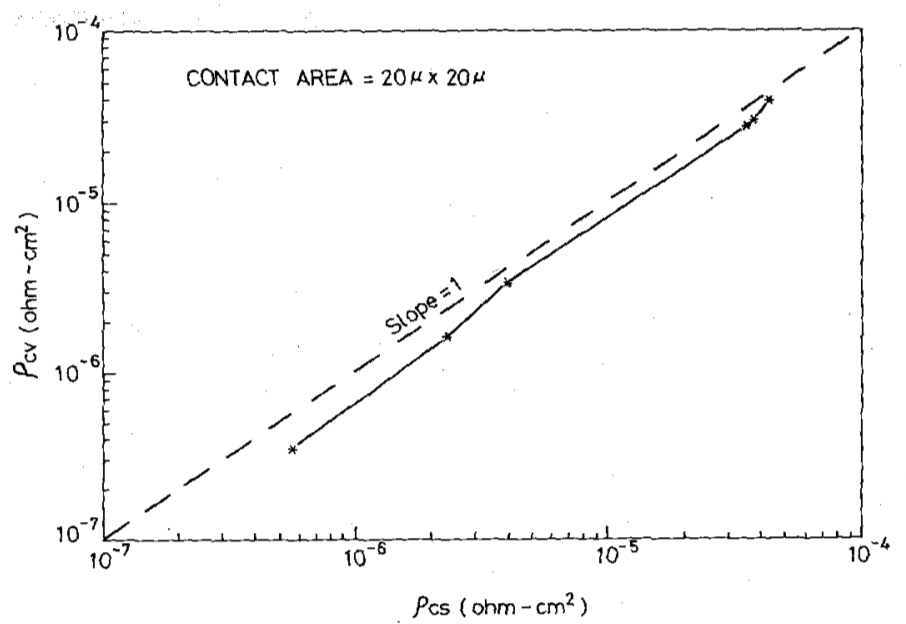

(a)

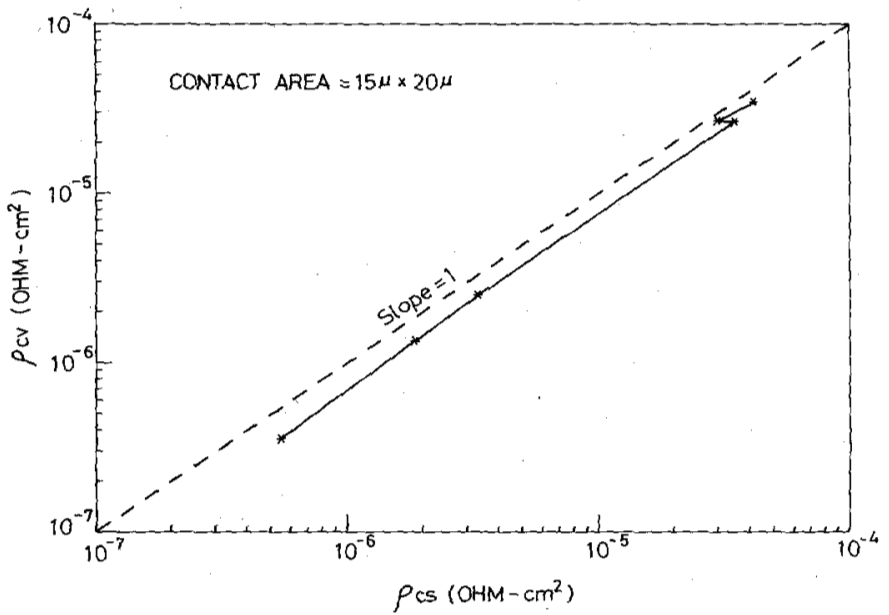

(b)

Fig. 5. The apparent specific contact resistivities of contact systems measured from the vertical structure $\rho_{c v}$ are plotted with those measured from the six-terminal structure $\rho_{c s}$. The contact area was kept constant for (a) $A_{c}=20 \mu \mathrm{m} \times 20 \mu \mathrm{m}$ and (b) $A_{c}=20 \mu \mathrm{m} \times 15 \mu \mathrm{m}$ during the measurement.

The $R_{s d}$ values obtained with the above method were much less than their corresponding $R_{s}$ values. Table I compiles the $R_{s d}$ values for five of the contacted wafers in this experiment along with their respective $R_{s}$ values. For wafers 1 and $3, R_{s d}$ were even one order of magnitude less than $R_{s}$. This result is consistent with that obtained by Reeves and Harrison [17] and Mazer and Lindholm [18].

Finally, the specific contact resistivities, measured by using the vertical Kelvin test structure, for these two metallization systems are plotted versus the surface implanted doses in Fig. 7. In these plottings, the contact areas were assumed to be uniform for the $\mathrm{Al}$ (1-percent $\mathrm{Si}) / \mathrm{TiSi}_{2} / \mathrm{Si}$ system during the computation of $\rho_{c}$. For both contact systems, $\rho_{c}$ drops nearly two orders of magnitude when the ion dose increases from $2 \times 10^{15} / \mathrm{cm}^{2}$ to $6 \times 10^{15} / \mathrm{cm}^{2}$. For the Al(1-percent Si) $/ \mathrm{Si}$ contact, $\rho_{c}$ is always less than that of the $\mathrm{Al}$ ( 1 -percent $\mathrm{Si}$ ) $/ \mathrm{TiSi}_{2} / \mathrm{Si}$ contact for the same ion dose. For the $\mathrm{Al}$ (1-percent $\mathrm{Si}) / \mathrm{TiSi}_{2}$ (direct reaction) $/ \mathrm{Si}$ system, a $\rho_{c}$ value of (1.17 


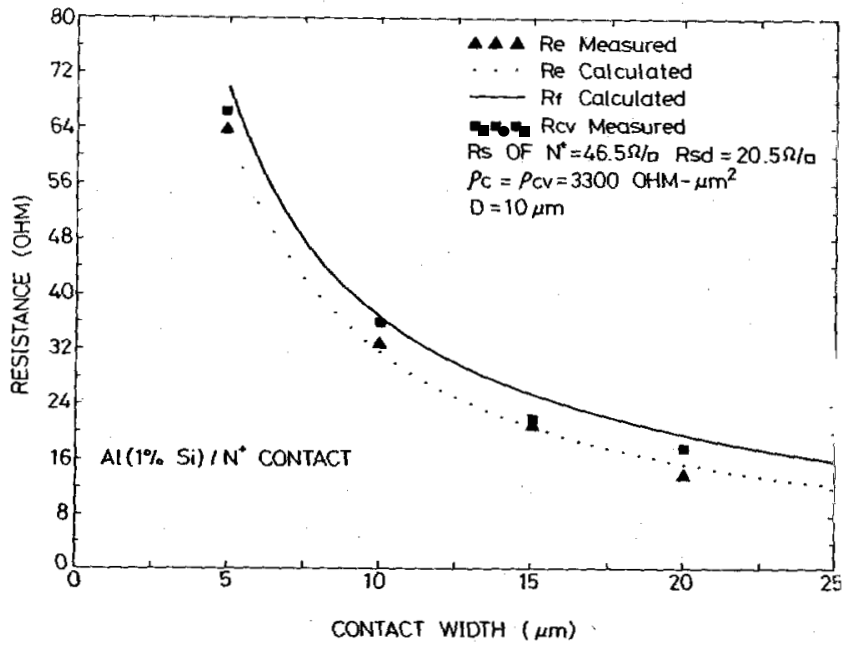

(a)

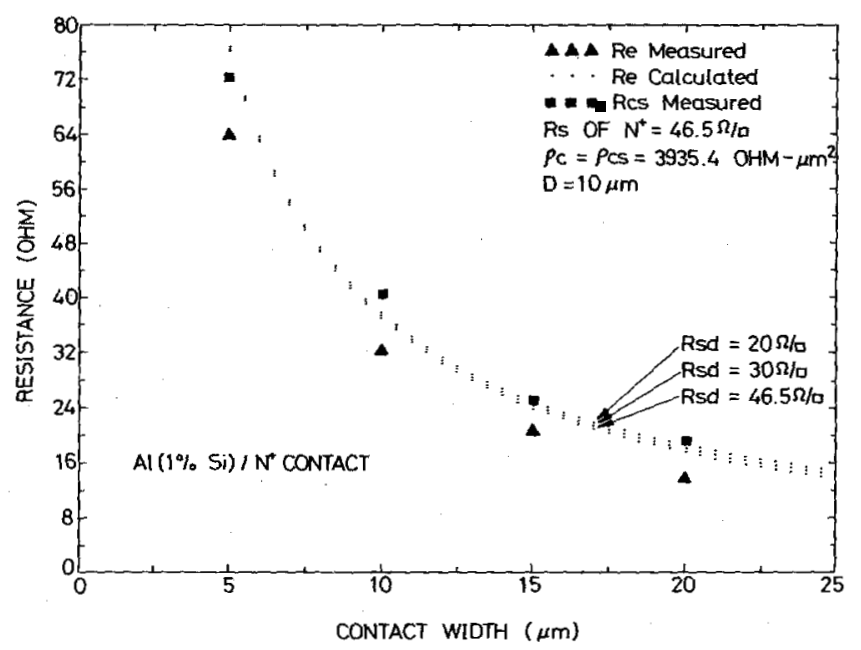

(b)

Fig. 6. (a) The measured $R_{c}, R_{e}$ and theoretical $R_{e}, R_{f}$ obtained from MTL equations with $\rho_{c}=\overline{\rho_{c v}}$ are plotted with different contact widths. The contact length in this case was kept at $10 \mu \mathrm{m}$. (b) $\overline{\rho_{c s}}$, instead of $\overline{\rho_{c v}}$, was used to calculate theoretical $R_{\varepsilon}$ and $R_{f}$ for the same wafer as (a). $R_{s d}$ was varied from 20 to $46.5 \Omega / \square$ and no good fit between measured $R_{e}$ and theoretical value can be obtaint:d.

TABLE I

The MEAsured $R_{s}$ AND $R_{s d}$ VAlues zOR SOME Of THE Fabricated Wafers

\begin{tabular}{|c|c|c|c|c|c|}
\hline Wafer No. & 1 & 2 & 3 & 4 & 5 \\
\hline Contact System & $A \perp\left(I \frac{\circ}{a} S i\right) / S i$ & $\mathrm{Al}(1 \% \mathrm{Si}) / \mathrm{Si}$ & $\begin{array}{l}\mathrm{Al}(\mathrm{l} \mathrm{g} \delta \mathrm{S}) / \mathrm{TiSi} \mathrm{i}_{2} / \mathrm{si} \\
\text { direct reaction }\end{array}$ & $\begin{array}{c}\mathrm{AI}(1 \% \mathrm{Si}) / \mathrm{TiSi}_{2} / \mathrm{Si} \\
\text { coevaporation }\end{array}$ & $\begin{array}{l}\mathrm{Al}(1 \circ \mathrm{Si}) / \mathrm{TiSi}_{2} / \mathrm{Si} \\
\text { direct reaction }\end{array}$ \\
\hline $\mathrm{R}_{\mathrm{Sd}}(\Omega, \square)$ & 1.29 & 20.5 & 1.96 & 9.27 & 13.3 \\
\hline $\mathrm{R}_{\mathrm{S}}(\Omega, \square)$ & 22.67 & 46.5 & 26.5 & 46.5 & 46.5 \\
\hline
\end{tabular}

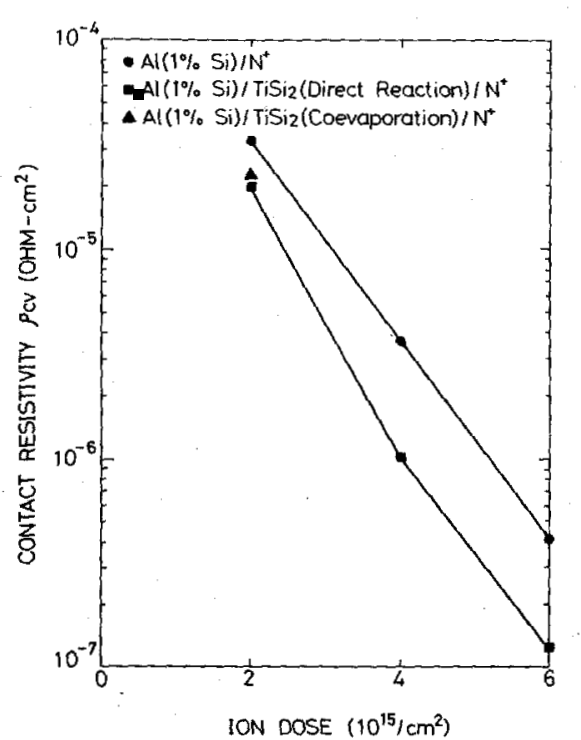

Fig. 7. The specific contact resistivities, measured by a vertical Kelvin test structure, of all the wafers are plotted versus the implanted doses of the conduction bars. $\pm 0.17) \times 10^{-7} \Omega \cdot \mathrm{cm}^{2}$ for a $5 \mu \mathrm{m} \times 5 \mu \mathrm{m}$ contact area with an implanted dose of $6 \times 10^{15} / \mathrm{cm}^{2}$ (corresponding to $N_{D}=1.69 \times 10^{20} / \mathrm{cm}^{3}$ ) was obtained. This $\rho_{c}$ value is compatible with the published data of $(1.7 \pm 0.2) \times$ $10^{-7} \Omega \cdot \mathrm{cm}^{2}$ (measured by the six-terminal method) at the same contact size and $N_{D}=1.9 \times 10^{20} / \mathrm{cm}^{3}$ [8]. In the experiments, it was also observed that the $\rho_{c}$ value for the $\mathrm{Al}$ (1-percent $\mathrm{Si}$ ) / $\mathrm{TiSi}_{2} / \mathrm{Si}$ system obtained by the direct-reaction method was always less than that obtained by the coevaporation method.

It should be mentioned that for this vertical Kelvin test structure, besides the interface nonuniformity, the errors mainly come from the misalignment of the first mask to form the isolation $\mathbf{p}-\mathbf{n}$ junction and the third mask to open the contact window. When this happens, the current flow is not strictly vertical at the periphery of the contact region. In experiments, it was found that, for some wafers, tine $R_{c v}$ as well as the $R_{c s}$ values obtained from one arm of the implanted bar were always larger than those obtained from the other arm. This indicated that a misalignment existed. Hence, averaging the four $R_{c v}$ values obtained by 
sensing on pad pairs $2-3,2-4,2-5$, and $2-6$ is a necessity. Other factors for causing errors in this test structure are the lateral diffusion and the depletion width of the isolation $p-n$ junction. However, these errors can be minimized by taking into account the lateral diffusion and the depletion width during the mask design.

\section{Conclusions}

From results depicted in Figs. 3-7, it can be concluded that the vertical Kelvin test structure does give better measurement on the specific contact resistivity. The current crowding effects and sheet resistance effect that are intrinsically inherent in other "horizontal"' test structures are eliminated if the misalignment and the lateral diffusion effects, which technically can be reduced to a minimum, are taken care of for this vertical test structure. If the contact interface is uniform, the measurement on the "true" specific contact resistivity becomes possible.

Also, in this work it was found that $\boldsymbol{R}_{s d}$ is much less than $R_{s}$. This confirms the results obtained in [17]. Finally, a specific contact resistivity of the value of $(1.17$ $\pm 0.17) \times 10^{-7} \Omega \cdot \mathrm{cm}^{2}$, measured by the vertical test structure, for the $\mathrm{Al}$ (1-percent $\mathrm{Si}$ ) $/ \mathrm{TiSi}_{2}$ (direct reaction ) / Si system, has been obtained.

\section{REPERENCES}

[1] J. Y.-T. Chen and D. B. Rensch, "The use of refractory metal and electron beam sintering to reduce contact resistance for VLSI," IEEE Trans. Electron Devices, vol. ED-30, pp. 1542-1550, Nov. 1983.

[2] S. Swirhum, K. C. Saraswat, and R. M. Swanson, "Contact resistance of LPCVD W/Al and PtSi/W/Al metallization," IEEE Electron Device Lett., vol. EDL-5, pp. 209-211, June 1984.

[3] S. S. Cohen, P. A. Piacente, G. Gildenblat, and D. M. Brown, "Platinum silicide obmic contacts to shallow junctions in silicon," J. Appl. Phys., vol. 53, no, 12, pp. 8856-8862, Dec. 1982.

[4] R. H. Cox and H. Strack, "Ohmic contacts for GaAs devices," SolidState Electron., vol. 10, pp. 1213-1218, 1967.

[5] W. Shockley, "Research and investigation of inverse epitaxial UHF power transistors," Air Force Atomic Lab., Wright-Patterson Air Force Base, OH, Rep. Al-TOR-207, Sept. 1964.

[6] S. S. Cohen, G. Gildenblat, M. Ghezzo, and D. M. Brown, "A1$1 \% \mathrm{Si} / \mathrm{Si}$ ohmic contacts to shallow junctions," J. Electrochem. Soc., vol. 129 , no. 6 , pp. $1335-1338,1982$.

[7] S. J. Proctor, L. W. Linholm, and J. A. Mazer, "Direct measurements of interfacial contact resistance, end contact resistance, and interfacial contact layer uniformity,' IEEE Trans. Electron Devices, vol. ED-30, pp. 1535-1541, Nov. 1983.

[8] M. Finetti, A. Scorzoni, and G. Soncini, "Lateral current crowding effects on contact resistance measurements in four terminal resistor test patterns," IEEE Electron Device Lett., vol. EDL-5, pp. 524526, Dec. 1984.

[9] W. H. Loh, K. Saraswat, and R. W. Dutton, "Analysis and scaling of Kelvin resistors for extraction of specific contact resistivity, "' IEEE Electron Device Lett., vol. EDL-6, pp. 105-108, Mar. 1985.

[10] T. F. Lei, L.-Y. Leu, and C. L. Lee, "A vertical Kelvin test structure for measuring the true specific contact resistivity," IEEE Electron Device Lett., vol. EDL-7, pp. 259-261, Apr. 1986.

[11] I. F. Chang, "Contact resistance in diffused resistors," J. Electrochem. Soc., vol. 117, pp. 368-372, Mar. 1970.

[12] P. Réveśz, J. Gyimesi, and 'E Zsoldos, "Growth of titanium silicide on ion-implanted silicon,' J. Appl. Phys., vol. 54, no. 4, pp. 1860 1864, Apr. 1983.

[13] M.-Z. Lin, private communication.
[14] H. K. Park, J. Sachitano, G. Eiclen, E. Lane, and T. Yamaguchi, "Mo/Ti bilayer metallization for a self-aligned TiSi, process," $J$, Vac. Sci. Technol., vol. A2, no. 2, pp. 259-263, April-June 1984.

[15] S. S. Cohen, "Contact resistance and methods for its determination," Thin Solid Films, vol. 104, pp. 361-379, 1983.

[16] T. F. Lei, C. L. Lee, J. H. Lin, and L. Y. Liau, "2-D analysis on specific contact resistance based on 4-terminal Kelvin resistor structure," in Proc. Int. EDMS (Republic of China), pp. 121-123, 1984.

[17] G. K. Reeves and H. B. Harrison, "Obtaining the specific contact resistance from transmission line model measurements," IEEE Electron Device Lett., vol. EDL-3, pp. 111-113, May 1982.

[18] J. A. Mazer and L. W. Linholm, "An improved test structure and Kelvin-measurement method for the determination of integrated circuit from contact resistance," J. Electrochem. Soc., pp. 440-443, Feb. 1985.

*

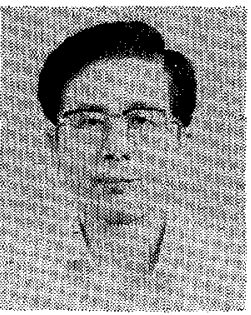

Tan Fu Lei was bom in Keelung, Taiwan, Republic of China, on September 17, 1944. He received the B.S. degree in electrical engineering from National Cheng Kung University, Tainan, Taiwan, in 1967 and the M.S. and Ph.D. degrees in electronic engineering from National Chiao Tung University, Hsinchu, Taiwan, in 1970 and 1979 , respectively.

From 1970 to 1972, he was with the Fine Products Microelectronics Corporation, Taiwan, as an Engineer working on the fabrication of small-signal transistors. From 1980 to 1982, he was the Plant Manager of Photronic Corporation, Taiwan, (now Silitek Corporation). Since 1983, he has been an Associate Professor in the Department of Electronics Engineering and the Institute of Electronics, National Chiao Tung University. During 1984 to 1986 , he was the Director of the Semiconductor Research Center, $\mathrm{Na}$ tional Chiao Tung University. His research interests are semiconductor devices and optoelectronics.

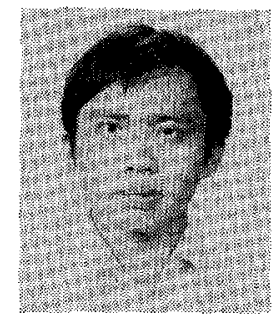

Len-Yi Leu was born on June 14, 1961, in Taiwan, Republic of China. He received the B.S. degree in electrophysics in 1983 and the M.S. degree in electronics, both from National Chiao Tung University. He is currently working toward the Ph.D. degree at the University of Southern California, Los Angeles. His research concerns the modeling of heterojunction interface characteristics.

His research interests include semiconductor MOS and CMOS devices in VLSI applications. devices, especially optoelectronic devices, and

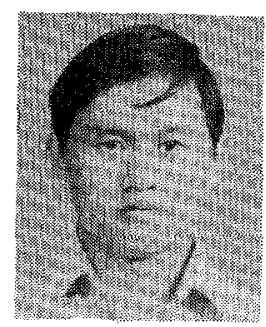

Chung Len Lee (S'71-M'81) was born in 1946 in Hu-Pei, China. He received the B.S. degree in electrical engineering from the National Taiwan University, Taiwan, Republic of China, in 1968, and the M.S. and Ph.D. degrees in electrical engineering from Carnegie-Mellon University, Pittsburgh, PA, in 1971 and 1975, respectively.

In 1975, he joined National Chiao Tung University as a faculty member, engaging in research on various topics on optoelectronics, semiconductor devices, integrated circuits, and processes. He has published more than 50 papers in the above areas. Presently, he is a Professor in the Department of Electronics Engineering at National Chiao Tung University.

Dr. Lee is a nember of Phi Kappa Phi and Phi Tau Phi. 